\title{
Localized surface optical phonon mode in the InGaN/GaN multiple- quantum-wells nanopillars: Raman spectrum and imaging
}

\author{
J. H. Zhu, ${ }^{1,2}$ J. Q. Ning, ${ }^{2}$ C. C. Zheng, ${ }^{2}$ S. J. Xu, ${ }^{2, a)}$ S. M. Zhang, ${ }^{1}$ and Hui Yang ${ }^{1,3, b)}$ \\ ${ }^{1}$ State Key Laboratory on Integrated Optoelectronics, Institute of Semiconductors, Chinese Academy of \\ Sciences, P. O. Box 912, Beijing 100083, China \\ ${ }^{2}$ Department of Physics and HKU-CAS Joint Laboratory on New Materials, The University of Hong Kong, \\ Pokfulam Road, Hong Kong, China \\ ${ }^{3}$ Suzhou Institute of Nano-tech and Nano-bionics, Chinese Academy of Sciences, Suzhou 215123, China
}

(Received 31 July 2011; accepted 29 August 2011; published online 16 September 2011)

An interesting phonon mode at around $685-705 \mathrm{~cm}^{-1}$ was clearly observed in the Raman spectra of InGaN/GaN multiple-quantum-wells nanopillars with different diameters at room temperature. The Raman peak position of this mode is found to show a distinct dependence on the nanopillar size, which is in well agreement with theoretical calculation of the surface optical (SO) phonon modes of nanopillars. Moreover, this kind of SO phonon was evidenced to be located on the pillar surface by using scanning confocal micro-Raman microscopy. (C) 2011 American Institute of Physics. [doi:10.1063/1.3640233]

The direct wide-bandgap gallium nitride $(\mathrm{GaN})$ and other III-nitride semiconductors have attracted a great deal of interest for almost 20 years since they have tremendous applications in many optoelectronic devices such as light-emitting diodes (LEDs) and blue laser diodes. ${ }^{1,2}$ Very recently, $\mathrm{GaN}$-based nanostructures, especially $\mathrm{InGaN} / \mathrm{GaN}$ multiplequantum-wells (MQW)-based nanostructures, have been fabricated and investigated owing to their increased internal quantum efficiencies and light extraction efficiencies. ${ }^{3-7}$

On the other hand, one interesting kind of lattice vibration can be localized on the surfaces of nanostructures, leading to a frequency intermediate between the transverse and longitudinal optical (TO and LO) phonon modes. ${ }^{8,9}$ Such phonon mode is usually called the surface optical (SO) phonon mode which could be observed when the translational symmetry of the surface potential is broken, i.e., surface roughness or construction of a grating. ${ }^{10}$ For the SO phonon modes, experimental observations have been reported in $\mathrm{GaP}$ and ZnS nanostructures. ${ }^{11-13}$ However, there are few reports on the SO phonon mode in the technologically important InGaN/ GaN MQWs structures which are commonly used as active layers in short-wavelength light-emitting devices. ${ }^{8,14-16}$

In this letter, we report an investigation of the SO phonon modes in the InGaN/GaN MQW nanopillars with different diameters by using scanning confocal micro-Raman spectroscopy/microscopy. The observed SO phonon frequency shows a distinct depend on the size of the nanopillars, which is consistent with the calculation result.

The GaN-based LED structures used in this study were grown by metalorganic chemical vapor deposition on c-axis sapphire substrates. Trimethylindium, trimethylgallium, and ammonia $\left(\mathrm{NH}_{3}\right)$ were used as indium, gallium, and nitrogen precursors in the epitaxy process, respectively. Bicyclopentadienyl magnesium $\left(\mathrm{Cp}_{2} \mathrm{Mg}\right)$ and silane $\left(\mathrm{SiH}_{4}\right)$ were employed as the sources of p-type and n-type dopants, respectively. The GaN-based LED structure has a $3.0 \mu \mathrm{m}$ n-type GaN:Si layer,

\footnotetext{
${ }^{a)}$ Electronic mail: sjxu@hkucc.hku.hk.

b)Electronic mail: hyang2006@sinano.ac.cn.
}

followed by five periods of InGaN/GaN MQW as an active layer, where well and barrier thicknesses were designed to be 3 and $30 \mathrm{~nm}$, respectively. After the deposition of MQW, a $0.2 \mu \mathrm{m}$ thick p-type GaN:Mg layer was deposited as the top contact layer. InGaN/GaN MQW nanopillars were then fabricated by using self-assembled Ni nanodots as etching mask. First, a $300 \mathrm{~nm}$ thick $\mathrm{SiO}_{2}$ thin film was deposited on the LED sample by plasma-enhanced chemical-vapor deposition, followed by the electron-beam evaporation of a $\mathrm{Ni}$ metal film. Then a rapid thermal annealing at $850^{\circ} \mathrm{C}$ under nitrogen ambient was used to form self-assembled Ni nanodots. Afterwards the sample was etched down to the n-type GaN layer with inductively coupled plasma etching technique and by using self-assembled Ni nanodots as etching mask to transfer the island pattern on the nano-sized $\mathrm{Ni}$ metal to the sample. Finally, the $\mathrm{Ni} / \mathrm{SiO}_{2}$ capping layer was removed by a buffer oxide etchant (BOE) to expose the MQW nanopillars. The nanopillar fabrication process has been reported in detail elsewhere. ${ }^{17}$ Nanopillars with different size and density can be obtained by using different Ni film thickness. From the scanning electron microscopy (SEM) images shown in Figs. 1 (a) -1 (d), we can see that the nanopillars with average diameters from $\sim 120$ to $250 \mathrm{~nm}$, respectively, and a height of approximately $700 \mathrm{~nm}$ were fabricated as the $\mathrm{Ni}$ film thickness was varied from 6 to $15 \mathrm{~nm}$.

The Raman spectra were recorded at room temperature using a WITec-Alpha scanning confocal micro-Raman system in a backscattering geometric configuration. The samples were excited with the $514.5 \mathrm{~nm}$ line of an $\mathrm{Ar}^{+}$laser. Figure 2 shows the Raman spectra collected from the unetched planar sample and nanopillar arrays with different diameters. Distinct peaks at about 569 and $734 \mathrm{~cm}^{-1}$ were observed for all the samples, which were assigned to the $\mathrm{E}_{2}$ (high) phonon and the $\mathrm{A}_{1}$ (LO) phonon mode of wurtzite GaN, respectively. ${ }^{18}$ In addition, a weak peak located around $417 \mathrm{~cm}^{-1}$ was also observed, which has been already identified to be a vibration mode of N-rich Ga-N bond configuration in GaN based nanostructures. ${ }^{18}$ As seen in Fig. 2, an additional peak around $685-705 \mathrm{~cm}^{-1}$ were observed for the 


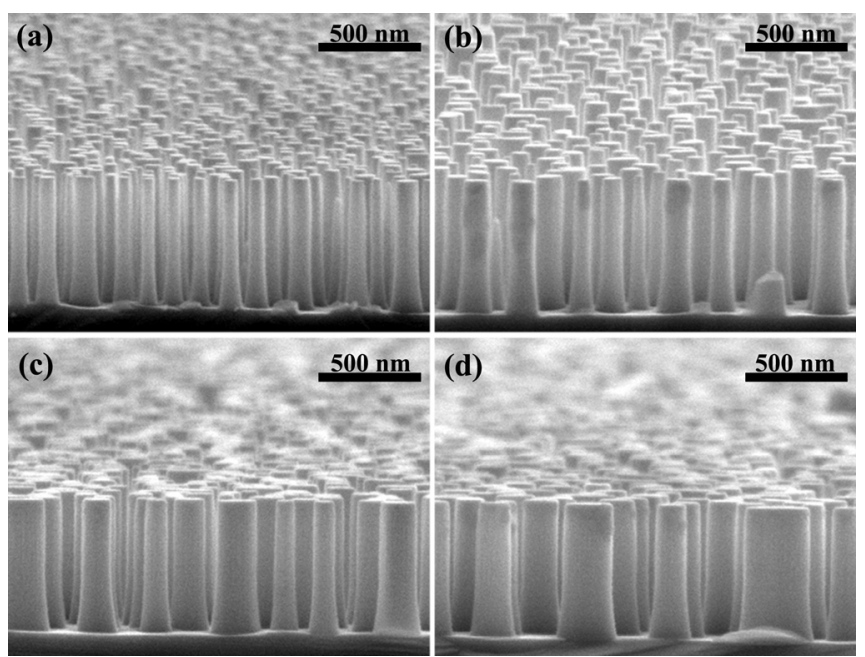

FIG. 1. The SEM images of the InGaN/GaN MQW nanopillars with various average diameters: (a) $120 \mathrm{~nm}$, (b) $160 \mathrm{~nm}$, (c) $200 \mathrm{~nm}$, (d) $250 \mathrm{~nm}$.

nanopillars compared with the unetched planar sample. Moreover, the Raman peak position of this additional mode depends strongly on the size of the InGaN/GaN MQW nanopillars, as marked by vertical arrows in Fig. 2. Due to the presence of surface roughness in the nanopillar sample and size dependence feature of the additional mode, we assign it to the SO phonon modes. From Fig. 2 the peak positions of the unique phonon mode of the nanopillars with average diameters of $120,160,200$, and $250 \mathrm{~nm}$ can be determined to be $687,692,699$, and $701 \mathrm{~cm}^{-1}$, respectively. Clearly, the phonon mode shifts toward the higher frequency direction with increasing the nanopillar diameter, indicating that the additional vibrational mode shall be the $\mathrm{SO}$ phonon mode in nanostructures.

It has been established that the SO phonon mode frequency is determined by the dielectric constant of the surrounding medium, as well as the particles shape and size..$^{9,10}$ In our case, the main parameter determining the SO phonon frequency is the diameter of the nanopillars. The expression for the SO phonon frequency from a 1-D nanostructure is given by ${ }^{9}$

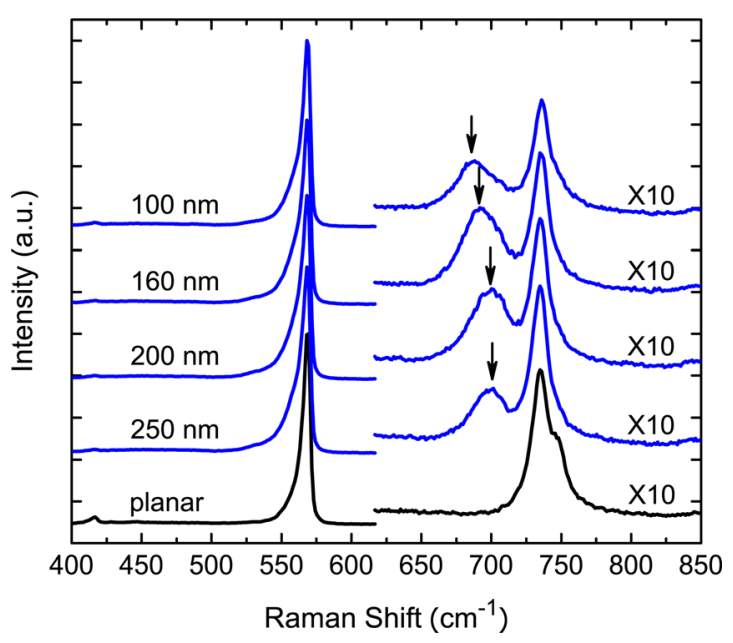

FIG. 2. (Color online) The room temperature Raman spectra of the InGaN/ GaN MQW nanopillars with average diameters varying from 120 to $250 \mathrm{~nm}$. An additional peak marked by vertical arrows was observed and shows a clear blueshift as the average diameter increases.

$$
\omega_{S O}^{2}=\omega_{T O}^{2} \frac{\varepsilon_{0}-\rho_{n x} \varepsilon_{m}}{\varepsilon_{\infty}-\rho_{n x} \varepsilon_{m}}
$$

where $\omega_{T O}$ is the TO phonon mode frequency, $\varepsilon_{0}$ and $\varepsilon_{\infty}$ the static and high-frequency dielectric constants of the material, respectively, and $\varepsilon_{m}$ the dielectric constant of the medium which is air in our case. And $\rho_{n x}$ is given by ${ }^{9}$

$$
\rho_{n x}=\frac{K_{0}^{\prime}(x) I_{0}(x)}{K_{0}(x) I_{0}^{\prime}(x)}=-\frac{K_{1}(x) I_{0}(x)}{K_{0}(x) I_{1}(x)}
$$

where $I_{j}(x)$ and $K_{j}(x)(\mathrm{j}=0,1)$ are the modified Bessel functions and $x=q \mathrm{r}$ ( $\mathrm{r}$ is the radius of the nanopillars and $q$ is the wave vector). The dielectric constant of the air $\varepsilon_{m}$ was taken as 1 . Values of $\varepsilon_{0}=10.3$ and $\varepsilon_{\infty}=6.7$ were used for $\operatorname{InN}$ and $\varepsilon_{0}=10.4$ and $\varepsilon_{\infty}=5.8$ for $\mathrm{GaN}$, respectively. ${ }^{14,16}$ Assuming that the In composition is 0.15 for our InGaN/GaN MQWs sample, the linear interpolate values of $\varepsilon_{0}$ and $\varepsilon_{\infty}$ are calculated to be 10.4 and 5.9 for $\operatorname{In}_{0.15} \mathrm{Ga}_{0.85} \mathrm{~N}$. As there are two TO phonons $\mathrm{A}_{1}(\mathrm{TO})$ and $\mathrm{E}_{1}(\mathrm{TO})$ in the wurtzite structure, in principle, two $\mathrm{SO}$ phonon modes should be expected. As $\mathrm{A}_{1}$ (TO) at $534 \mathrm{~cm}^{-1}$ and $\mathrm{E}_{1}$ (TO) at $561 \mathrm{~cm}^{-1}$ were used for $\mathrm{GaN}$ and $\mathrm{A}_{1}$ (TO) at $447 \mathrm{~cm}^{-1}$ and $\mathrm{E}_{1}$ (TO) at $478 \mathrm{~cm}^{-1}$ for InN, ${ }^{14,16}$ the $A_{1}(\mathrm{TO})$ and $\mathrm{E}_{1}(\mathrm{TO})$ in $\operatorname{In}_{0.15} \mathrm{Ga}_{0.85} \mathrm{~N} / \mathrm{GaN}$ MQWs were calculated to be 521 and $549 \mathrm{~cm}^{-1}$, respectively. It is known that only SO phonon mode pertaining to $\mathrm{E}_{1}$ (TO) character can be distinctly observed for InGaN by Raman scattering. ${ }^{16}$ So the observed SO phonon peak in our experiment shall be associated with $\mathrm{E}_{1}$ mode. Figure 3(a) shows the $\mathrm{E}_{1}$ related $\omega_{S O}$ as a function of $q \mathrm{r}$ with Eqs. (1) and (2). A horizontal line indicates the experimental SO phonon frequency $\left(\omega_{S O}=687 \mathrm{~cm}^{-1}\right)$ observed by Raman
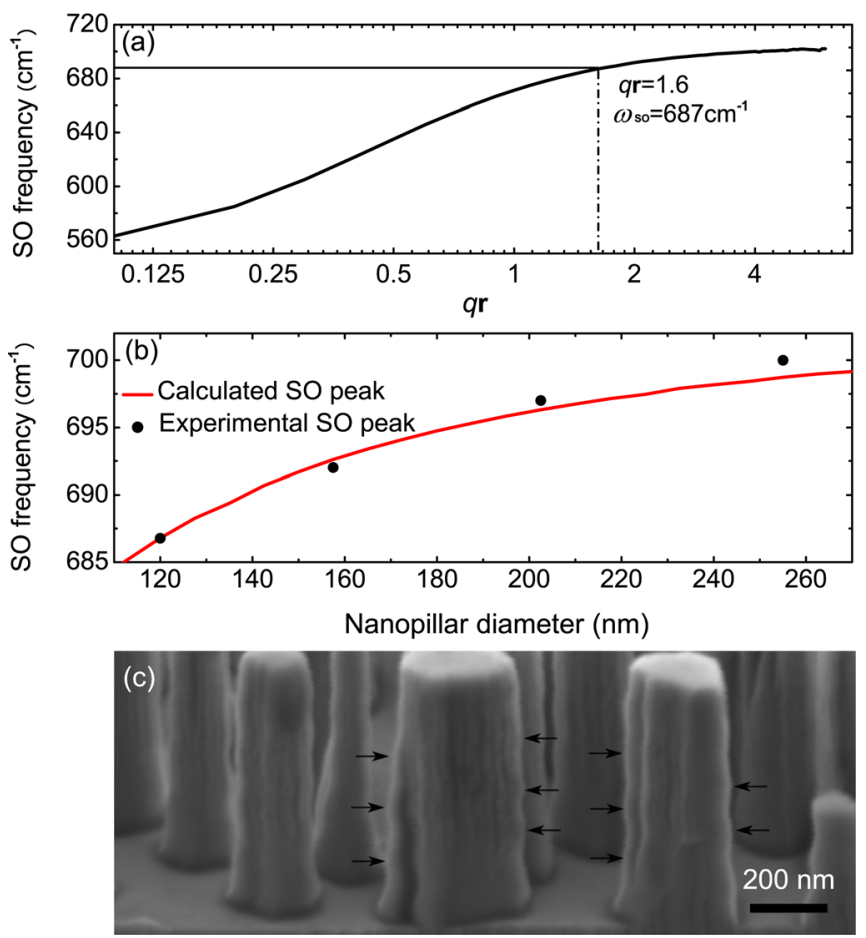

FIG. 3. (Color online) (a) Calculated SO phonon modes of InGaN/GaN nanopillars as a function of $q r$. (b) The measured and calculated Raman SO modes for nanopillars with different diameters. (c) Surface roughness in typical InGaN/GaN MQW nanopillars. Arrows show the modulation in the sidewall surface of nanopillars. 
TABLE I. The adopted values of $q \mathrm{r}$, calculated and measured Raman SO mode for the InGaN/GaN MQW nanopillars with different average diameters.

\begin{tabular}{llccc}
\hline \hline Nanopillar diameter $(\mathrm{nm})$ & 120 & 160 & 200 & 250 \\
\hline$q \mathrm{r}$ & 1.60 & 2.13 & 2.67 & 3.33 \\
Calculated peak $\left(\mathrm{cm}^{-1}\right)$ & 687.0 & 692.6 & 697.0 & 699.0 \\
Measured peak $\left(\mathrm{cm}^{-1}\right)$ & 687.0 & 692.0 & 698.0 & 700.0 \\
\hline \hline
\end{tabular}

scattering for the nanopillars with diameter of $120 \mathrm{~nm}$. Then, the corresponding wave vector q can be calculated as shown in Fig. 3(a) (for $\mathrm{r}=\mathrm{d} / 2=60 \mathrm{~nm}, \omega_{S O}=687 \mathrm{~cm}^{-1}, q \mathrm{r}=1.6$, $q \approx 0.027 \mathrm{~nm}^{-1}$ ). As the nanopillars with different diameters were fabricated with the same process and the undulation period in the sidewall surface for different diameters was similar as shown in Fig. 3(c), $q \approx 0.027 \mathrm{~nm}^{-1}$ can be taken for all the nanopillars. Then the values of $q \mathrm{r}$ were calculated to be 2.13, 2.67, and 3.33 for the InGaN/GaN MQW nanopillars with diameters of 160,200 , and $250 \mathrm{~nm}$, respectively. The values of $q \mathrm{r}$, experimental and calculated SO phonon mode peaks for different diameters are listed in Table I and plotted in Fig. 3(b) for comparison. From Fig. 3(b) it can be seen that good agreement between experiment and calculation has been achieved.

The SO phonon intensity is determined by the magnitude of surface roughness due to the breakdown of translation symmetry of the surface potential. ${ }^{12}$ Therefore, the wavelength of the surface potential which is responsible for breaking the symmetry and activating the SO phonon was then evaluated by $\lambda=2 \pi / q$ and calculated to be $236 \mathrm{~nm}$ when $q=0.027 \mathrm{~nm}^{-1}$ for the InGaN/GaN MQW nanopillars. As shown in Fig. 3(c), surface roughness with an undulation period of $\sim 120 \mathrm{~nm}$ was observed in nanopillars. Modulation period with any integral multiple equivalent to $\lambda$ is sufficient to initiate breakdown of translational symmetry for the surface potential contribution to the SO phonons. In our case, 2 $\mathrm{nm} \times 120 \mathrm{~nm}=240 \mathrm{~nm}$ which is approximately equal to $\lambda$ can make the intensity of the surface modes comparable to those of other observed phonons modes.

Because the SO phonon mode is localized to the surface and interface, ${ }^{10}$ the SO phonon intensity should be associated with the surface configuration of the nanopillars. We have measured the Raman images of the nanopillar and unetched planar samples for an area of $3 \mu \mathrm{m} \times 3 \mu \mathrm{m}$. The images of $\mathrm{GaN} \mathrm{E}_{2}$ (high)-mode integral intensity for the planar sample, $\mathrm{GaN} \mathrm{E}_{2}$ (high)-mode and the $\mathrm{SO}$ phonon mode integral intensity for the $160 \mathrm{~nm}$ nanopillars are shown in Figs. 4(a)-4(c) for comparison. In sharp contrast to the homogeneous distribution of the $\mathrm{GaN}_{2}$ (high)-mode in the planar sample, the Raman images of the nanopillars clearly exhibit the pillar distribution with bright spots. More interestingly, the Raman images of the $\mathrm{GaN} \mathrm{E}_{2}$ (high)-mode and the SO mode in the nanopillar sample show a distinct spatial correlation, indicating that the SO phonon modes are really localized on the nanopillar's surface.

In conclusion, the InGaN/GaN MQW nanopillars with different diameters using self-assembled $\mathrm{Ni}$ nanodots as

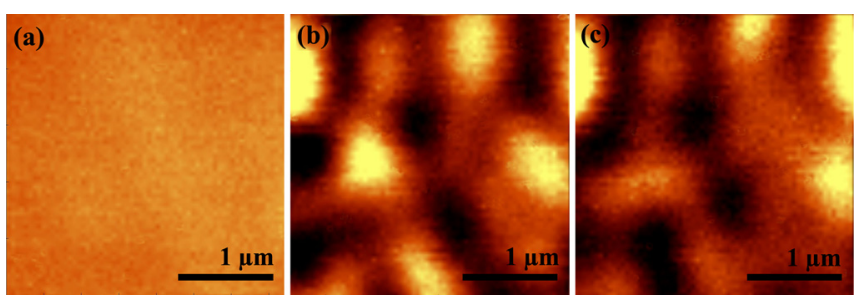

FIG. 4. (Color online) The integral intensity Raman images: (a) for the GaN $\mathrm{E}_{2}$ (high) mode of the planar sample; (b) for the $\mathrm{GaN} \mathrm{E}_{2}$ (high) mode of the $160 \mathrm{~nm}$ nanopillars; (c) for the SO phonon mode of the $160 \mathrm{~nm}$ nanopillars.

etching mask were fabricated. An interesting SO phonon mode was firmly identified in the nanopillars through Raman spectroscopic and imaging measurements and theoretical calculations. The characteristic frequency of the SO phonon mode was found to show a theoretically predicted dependence on the diameter of nanopillars. Spatially correlated relationship between the Raman images for $\mathrm{GaN}$ mode and $\mathrm{SO}$ mode was observed, giving an additional support to the conclusion that the SO phonon modes are localized on the surfaces of nanopillars.

The work described in this paper was supported by the Joint Research Fund for Overseas Chinese, Hong Kong and Macau Scientists of NSFC (Grant No. 61028012) and the National Basic Research Program of China (Grant No. 2007CB936701).

${ }^{1}$ S. Nakamura, M. Senoh, S. Nagahama, N. Iwasa, T. Yamada, T. Matsushita, H. Kiyoku, and Y. Sugimoto, Jpn. J. Appl. Phys. 35, L74 (1996).

${ }^{2}$ S. Nakamura, M. Senoh, S. Nagahama, N. Iwasa, T. Yamada, T. Matsushita, H. Kiyoku, Y. Sugimoto, T. Kozaki, H. Umemoto, M. Sano, and K. Chocho, Jpn. J. Appl. Phys. 37, L309 (1998).

${ }^{3}$ H. M. Kim, Y. H. Cho, H. Lee, S. I. Kim, S. R. Ryu, D. Y. Kim, T. W. Kang, and K. S. Chung, Nano Lett. 6, 1059 (2004).

${ }^{4}$ L. B. Chang, S. S. Liu, and M. J. Jeng, Jpn. J. Appl. Phys. 40, 1242 (2001).

${ }^{5}$ C. H. Chiu, T. C. Lu, H. W. Huang, C. F. Lai, C. C. Kao, J. T. Chu, C. C. $\mathrm{Yu}$, H. C. Kuo, S. C. Wang, C. F. Lin, and T. H. Hsueh, Nanotechnology 18, 445201 (2007).

${ }^{6}$ C. J. Neufeld, C. Schaake, M. Grundmann, N. A. Fichtenbaum, S. Keller, and U. K. Mishra, Phys. Stat. Sol. (c) 4, 1605 (2007).

${ }^{7}$ H. W. Choi, M. D. Dawson, P. R. Edwards, and R. W. Martin, Appl. Phys. Lett. 83, 4483 (2003).

${ }^{8}$ X. J. Wang, L. L. Wang, W. Q. Huang, L. M. Tang, B. S. Zou, and K. Q. Chen, Semicond. Sci. Technol. 21, 751 (2006).

${ }^{9}$ R. Ruppin and R. Englman, Rep. Prog. Phys. 33, 149 (1970).

${ }^{10}$ B. E. Sernelius, Surface Modes in Physics (Wiley, New York, 2001).

${ }^{11}$ Z. C. Zhang and Q. X. Zhang, J. Nanopart. Res. 12, 961 (2010).

${ }^{12}$ K. W. Adu, Q. Xiong, H. R. Gutierrez, G. Chen, and P. C. Eklund, Appl. Phys. A: Mater. Sci. Process 85, 287 (2006).

${ }^{13}$ R. Gupta, Q. Xiong, G. D. Mahan, and P. C. Eklund, Nano Lett. 3, 1745 (2003).

${ }^{14}$ C. L. Hsiao, L. W. Tu, T. W. Chi, M. Chen, T. F. Young, C. T. Chia, and Y. M. Chang, Appl. Phys. Lett. 90, 043102 (2007).

${ }^{15}$ S. Sahoo, M. S. Hu, C. W. Hsu, L. C. Chen, K. H. Chen, A. K. Arora, and S. Dhara, Appl. Phys. Lett. 93, 233116 (2008).

${ }^{16}$ S. E. Wu, S. Dhara, T. H. Hsueh, Y. F. Lai, C. Y. Wanga, and C. P. Liua, J. Raman Spectrosc. 40, 2044 (2009).

${ }^{17}$ J. H. Zhu, S. M. Zhang, X. Sun, D. G. Zhao, J. J. Zhu, Z. S. Liu, D. S. Jiang, L. H. Duan, H. Wang, Y. S. Shi, S. Y. Liu, and H. Yang, Chin. Phys. Lett. 25, 3485 (2008).

${ }^{18}$ J. Q. Ning, S. J. Xu, D. P. Yu, Y. Y. Shan, and S. T. Lee, Appl. Phys. Lett. 91, 103117 (2007). 